\title{
Action de la diéthylcarbamazine sur les microfilaires de Dipetalonema dessetae chez son hôte définitif
}

\author{
par Ph. GAYRAL (*), M. C. PFAFF (**), G. MAHUZIER (**), \\ F. PRADEAU (*) et M. LECOUSTILLIER (*) \\ Laboratoires de Parasitologie (*) et de Chimie analytique (**), \\ Faculté de Pharmacie, F 92290 Châtenay-Malabry.
}

\section{Résumé.}

Les auteurs utilisent une filaire de Rongeurs à microfilarémie périodique diurne dont les adultes et les microfilaires sont détruits par la diéthylcarbamazine (DEC). A l'inverse de ce que l'on observe avec d'autres filaires périodiques, une administration orale nocturne de DEC entraîne non une augmentation brutale et brève de la microfilarémie, mais une chute de près de $70 \%$ en $15 \mathrm{mn}$. Ceci peut être dû à une absorption digestive très rapide de la DEC, mise en évidence par des concentrations sériques maximales atteintes en $15 \mathrm{mn}$.

\section{Summary.}

Microfilaricidal action of Diethylcarbamazine on Dipetalonema dessetae in his natural host.

With a Filaria of Rodent whose microfilariae are periodic and diurnal, and destroyed by DEC, the authors don't observe the immediate rise of microfilaria count sometimes noted following treatment of periodic filaria during the low phase of microfilaremia. Moreover, the microfilaremia falls at $30 \%$ of the pre-treatment level after $15 \mathrm{mn}$. This may be in relation with the swift digestive absorption, as detected by the rise of the DEC serum level, whose maximum is reached within $15 \mathrm{mn}$.

\section{Introduction}

Le mode d'action de la diéthylcarbamazine (DEC) sur les microfilaires sensibles à ce médicament n'est pas élucidé. Habituellement, son activité est extrêmement brutale et se traduit par une chute importante de la microfilarémie, dès les premières heures,

Accepté le 13 juin 1978. 
ou parfois les minutes qui suivent son administration. Avec certaines microfilaires présentant une périodicité, cette action est différente lorsque l'administration de la DEC se fait en phase de microfilarémie minimum, une augmentation brutale et temporaire précède cette chute de la microfilarémie. Ce phénomène serait dû à la libération, sous l'action du médicament, des microfilaires séquestrées dans les capillaires pulmonaires où elles se tiendraient lorsqu'elles ne sont pas dans le sang périphérique (Hawking et Adams, 1964).

Ainsi, chez le chien parasité par Dirofilaria immitis, Fukamashi, 1960 (in Hawking et Adams, 1964) signale une augmentation de la microfilarémie persistant 3 heures après le traitement mais pouvant être supprimée par l'atropine ou par section du pneumogastrique. Desowitz et al. (1975) notent aussi cet effet stimulant de la DEC, 15 à 20 minutes après traitement des chiens à $10 \mathrm{mg}$ par $\mathrm{kg}$.

Chez l'homme adulte parasité par une souche à périodicité nocturne de Wuchereria bancrofti, Hawking et Adams (1964) administrent de jour $100 \mathrm{mg}$ de DEC par voie intraveineuse et observent une augmentation de la microfilarémie 2 minutes après l'injection, persistant plus de 30 minutes, sans inhibition par l'atropine. De même, Sullivan et Hembree (1970) détectent les microfilaires une heure après un traitement par voie orale à $200 \mathrm{mg}$ le matin.

Dans les filarioses expérimentales à microfilarémie non périodique, cette action n'est pas retrouvée, même chez celles dont les microfilaires sont détruites par la DEC. C'est le cas de Litomosoides carinii parasite de Sigmodon hispidus (Hawking et al., 1950) ou de Brugia pahangi chez le chat (Denham et al., 1971).

Or, nous disposons d'une Filaire expérimentale de Rongeurs, dont les microfilaires sont très sensibles à ce médicament et dont la microfilarémie est périodique. Ceci nous donne la possibilité de rechercher ce comportement particulier des microfilaires après une administration de DEC en période de microfilarémie basse.

En outre, pour mettre en évidence une éventuelle liaison entre cette stimulation des microfilaires, l'action microfilaricide et la concentration sérique en DEC, nous avons dosé ce produit dans le sang, lors de chaque comptage de la microfilarémie.

\section{Matériel et méthodes}

Dipetalonema dessetae est une Filaire parasite de Proechimys oris, Rongeur Caviomorphe brésilien (Bain, 1973). Les adultes vivent dans la cavité péritonéale du Rongeur, les microfilaires dépourvues de gaine sont sanguicoles. En laboratoire, la transmission s'effectue avec Aedes aegypti. La sensibilité des Filaires adultes et des microfilaires à de nombreux antifilariens comme la DEC, le lévamisole, le trichlorfon, la suramine en fait un modèle utilisable dans l'étude de leur mode d'action et la recherche de nouveaux filaricides (Gayral et Pommiès, 1976).

La microfilarémie est périodique diurne avec un maximum de 08 à $16 \mathrm{~h}$, un minimum nocturne de $20 \mathrm{~h}$ à $04 \mathrm{~h}$, correspondant au quart des valeurs diurnes. Cette périodicité a été déterminée en octobre 1976 sur 3 Proechimys dont les microfilarémies 
étaient à 12 heures respectivement de 97,188 et 1178 microfilaires pour $10 \mathrm{~mm}^{3}$ de sang, et en mai 1977 sur 4 autres dont les microfilarémies étaient à 12 heures de 19 , 68,74 , et 216 pour $10 \mathrm{~mm}^{3}$ de sang. Des numérations de microfilaires ont été effectuées toutes les 4 heures. La figure 1 donne les variations des pourcentages par rapport au $100 \%$ de $12 \mathrm{~h}$ (avec l'intervalle de confiance de la moyenne). Tous les horaires sont en GMT +1 (heure d'hiver).

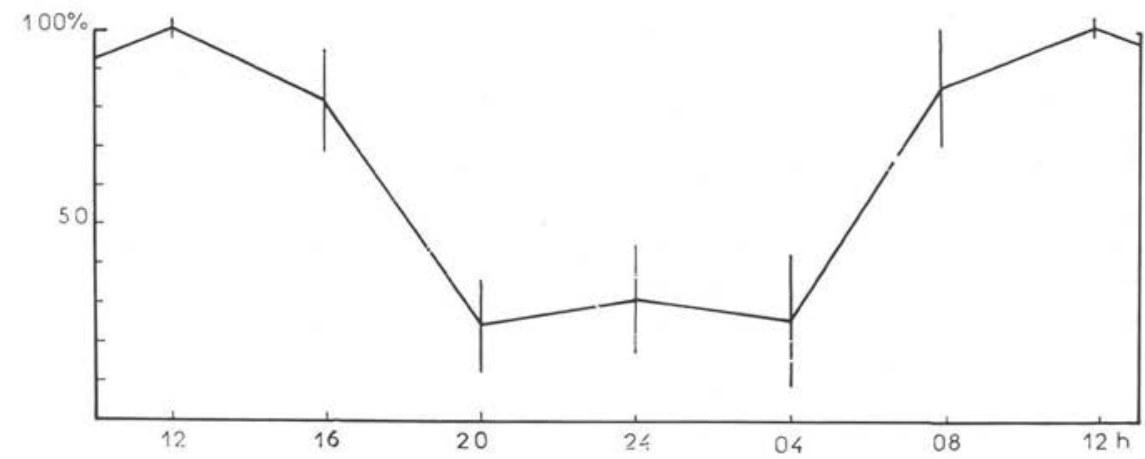

Fig. 1. Périodicité de la microfilarémie chez Proechimys oris parasité par Dipetalonema dessetae (temps: GMT+1) : moyenne des pourcentages par rapport aux maximums diurnes.

Pour cette étude de l'action de la DEC, les Proechimys sont traités par voie orale à une dose unique de $100 \mathrm{mg} / \mathrm{kg}$ en base. On utilise une solution aqueuse de citrate (Notézine N.D. SPECIA). Ils sont maintenus à la diète hydrique 10 à 12 heures avant le traitement. Les prélèvements sanguins au sinus orbitaire sont effectués au temps 0 (to), puis à $15,30,60,120,180$ minutes et éventuellement 12 ou 24 heures.

La microfilarémie est comptée sur une ou plusieurs gouttes épaisses calibrées et elle est toujours ramenée à un volume de $10 \mathrm{~mm}^{3}$ de sang. La DEC sérique est dosée par chromatographie en phase gazeuse, selon une méthode que nous avons décrite précédemment (Pfaff et al., 1978).

\section{Résultats}

\section{Essai de jour (tableau I) :}

Le Proechimys n $\mathrm{n}^{\circ} 339$ est un mâle âgé de 280 jours pesant $210 \mathrm{~g}$. La microfilarémie était de 61 microfilaires avant le traitement à $08 \mathrm{~h}$. Elle chute de $60 \%$ en 15 minutes, puis progressivement jusqu'à $92 \%$ après 3 heures. Elle est de $13 \%$ à 24 heures et remonte pour rester à $40-50 \%$ avec un recul de 5 mois environ. 
Tableau I. Microfilarémies après une dose orale de $100 \mathrm{mg} / \mathrm{kg}$ de jour (traitement effectué à $8 \mathrm{~h}$ ).

$\left.\begin{array}{c|c|c|c|c|c|c|c}\hline 339 & \text { to } & 15 \mathrm{mn} & 30 \mathrm{mn} & 1 \mathrm{~h} & 2 \mathrm{~h} & 3 \mathrm{~h} & 24 \mathrm{~h} \\ \hline \text { Microfilarémie } & 61 & 23 & 26 & 23 & 18 & 5 & 8 \\ \hline \% \text { par rapport à to } & 100 & 38 & 42 & 38 & 30 & 8 & 13\end{array}\right\}$

\section{Essai de nuit (tableau II et fig. 2) :}

Deux Proechimys femelles $\mathrm{n}^{\circ} 337$ et 340 âgées de 360 jours, pesant environ $200 \mathrm{~g}$ ont été traitées à $21 \mathrm{~h} 45$. La microfilarémie du 337 était de 75 microfilaires de jour et, la nuit, de 28 avant le traitement, soit $37 \%$ du maximum. Celle du 340 était de 400 de jour et 152 de nuit, avant le traitement, soit $38 \%$ du maximum diurne.

Tableau. II. Microfilarémies et concentrations sériques en DEC après un dose orale de $100 \mathrm{mg} / \mathrm{kg}$ de nuit (traitements effectuées à $21 \mathrm{~h} \mathrm{45)}$.

\begin{tabular}{|c|c|c|c|c|c|c|c|c|}
\hline & de jour & $t_{0}$ & $15 \mathrm{mn}$ & 30 an & $1 \mathrm{~h}$ & $2 \mathrm{~h}$ & $3 \mathrm{~h}$ & $12 \mathrm{~h}$ \\
\hline$\frac{337}{\text { Microfilarémie }}$ & 75 & 28 & 12 & 9 & 8 & 8 & 3 & 2 \\
\hline$\%$ par rapport à to & & $100 \%$ & 43 & 32 & 28 & 28 & 11 & 7 \\
\hline DEC en $\mathrm{mg} / \mathrm{l}$ & & 0 & 17 & 14,65 & 7,8 & 3,3 & 1,78 & 0 \\
\hline$\frac{340}{\text { Microfilarémie }}$ & 400 & 152 & 39 & 35 & - & - & 18 & 33 \\
\hline$\%$ par rapport à $t_{0}$ & & $100 \%$ & 25 & 23 & & & 12 & 22 \\
\hline $\mathrm{DEC}$ en $\mathrm{mg} / \mathrm{l}$ & & 0 & 28,6 & 16,5 & 10,2 & 2,32 & 0,3 & 0 \\
\hline
\end{tabular}


Après traitement, la microfilarémie chute en 15 minutes de $57 \%$ pour le premier Proechimys, de $75 \%$ pour le second, et en 3 heures de $90 \%$ pour les deux. Ensuite, elle ne regagne pas son niveau d'avant traitement, et persiste à $40-50 \%$ du maximum diurne.

En ce qui concerne la DEC, après une absorption de $20 \mathrm{mg}$ par animal, on constate que les deux cinétiques sont très voisines avec un maximum vers 15 minutes. L'élimination est régulière et rapide avec des demi-vies de l'ordre de 40 minutes (fig. 2).

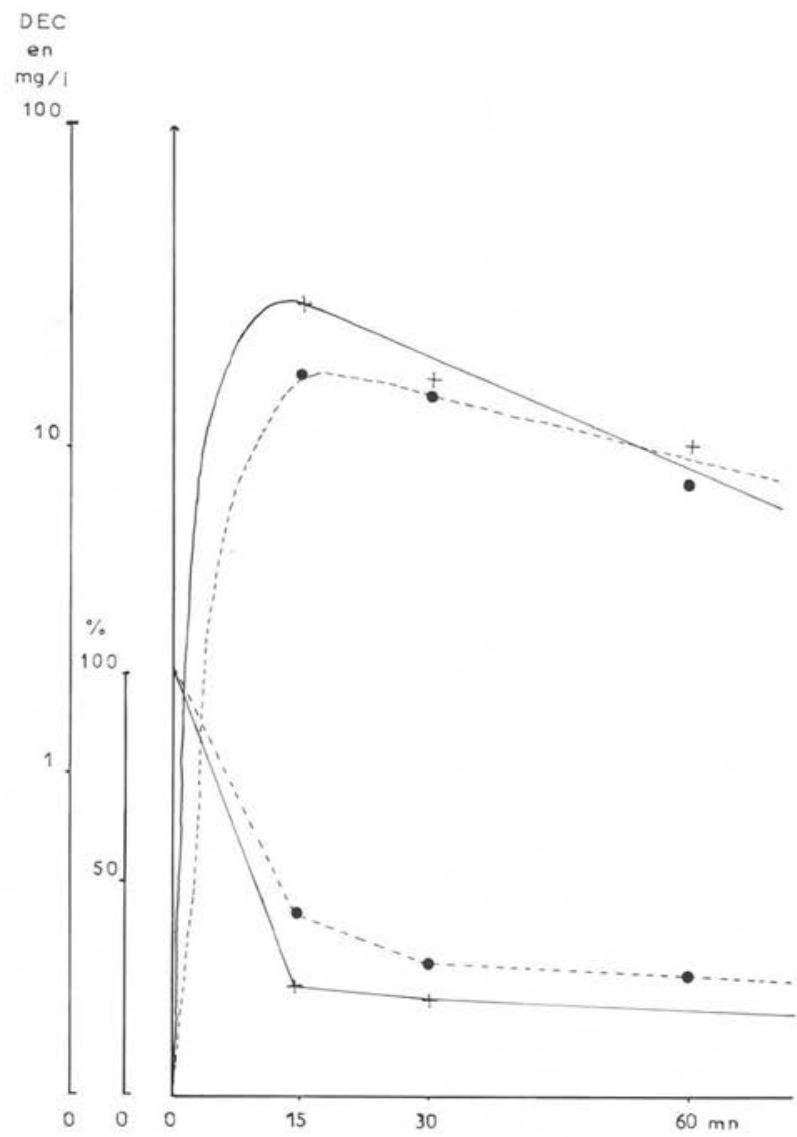

Fig. 2. Traitement à la DEC nocturne: variations de la microfilarémie en pourcentage des valeurs observées avant traitement (to). Taux sériques de DEC en mg/l. $\mathrm{n}^{\circ} 340$ 


\section{Discussion}

L'activité microfilaricide de la DEC est rapide et intense, de jour comme de nuit. On n'observe pas de «stimulation» de la microfilarémie avant la réduction due au traitement. Cette remontée temporaire est observée dans certaines filarioses dont les microfilaires sont périodiques et Hawking et Adams (1964) l'attribuent à une « chasse » des microfilaires vers le sang périphérique à partir des poumons, où elles se concentreraient pendant la phase de microfilarémie minimale.

Bien que les microfilaires de $D$. dessetae soient périodiques, on n'observe donc pas ce comportement et pour expliquer cette différence, on peut envisager plusieurs hypothèses. Les microfilaires de $D$. dessetae peuvent se cantonner la nuit dans d'autres organes que le poumon où elles seraient moins sensibles au médicament. Ou bien la fixation tissulaire de la DEC peut varier selon les hôtes, et n'être pas la même chez l'Homme et le Proechimys. Cette hypothèse semble confortée par les premières données de pharmacocinétique obtenues chez le Proechimys (résultats non publiés).

D'autre part, l'activité de la DEC est rapide puisque 15 minutes après l'administration par voie orale, la microfilarémie a déjà chuté de 50 à $75 \%$. En fait, ceci découle de l'absorption digestive extrêmement rapide de la DEC mise en évidence par une concentration maximale sérique atteinte dès 15 minutes.

Nous avons précédemment signalé l'activité remarquable de la DEC sur les microfilaires, mais aussi sur les adultes de D. dessetae chez le Proechimys (Gayral et Pommiès, 1976). Elle semble être due à la sensibilité propre des parasites, mais surtout à un métabolisme original de l'hôte que nous explorons actuellement.

\section{Bibliographie}

Bain O., 1973 : Une nouvelle Filaire de Rongeur sud-américain Dipetalonema dessetae, n. sp. (Nematoda, Filarioidea). Bull. Mus. Nat. Hist. Nat., 3e série, 116, Zool. 90, 309-316.

Denham D.A., Ponnudurai T., Nelson G.S., Guy F.,Rogers R., 1971: The effect of metrifonate on Brugia pahangi infections in domestic cats. Bull. Wld Hlth Org., 45, 423-429.

Desowitz R.S., Palumbo N., Read G., Una S.R., 1975: The nature of the adverse reaction to DEC treatment in dogs infected with Dirofilaria immitis. Trans. R. Soc. Trop. Med. Hyg., 69, 430.

Gayral Ph., Pommiès M., 1976: Résultats préliminaires sur l'utilisation d'une nouvelle Filaire de Rongeur Dipetalonema dessetae (Bain, 1973) dans l'évaluation des antifilariens. C.R. Acad. Sci., série D, 183, 861-864.

Hawking F., Sewell P., Thurston J.P., 1950: The mode of action of Hetrazan on filarial worms. $\mathrm{Br}$. J. Pharmacol., 5, 217-238.

Hawking F, Adams W.E., 1964 : Microfilaricidal action of diethylcarbamazine in vivo: first phase. Ann. Soc. Belge Med. Trop., 44, 279-284.

Pfaff M.C.. Gayral Ph., Mahuzier G., 1978: Méthode de dosage par chromatographie en phase gazeuse de la diéthylcarbamazine (Hétrazan) dans le sérum de rongeurs de laboratoire. J. Chromat., 150, 155-160.

Sullivan T.J., Hembree S.C., 1970: Enhancement of the density of circulating microfilariae with diethylcarbamazine. Trans. R. Soc. Trop. Med. Hyg., 64, 787-788. 